\title{
Green and Metal-free Catalytic Oxidation of Urazoles into Triazolinediones by Guanidinium Nitrate and Catalytic Amounts of Silica Sulfuric Acid
}

\author{
Mohsen Nikoorazm, ${ }^{*}$ Arash Ghorbani-Choghamarani, ${ }^{*}$ Hamid Goudarziafshar, and Shadpour Mallakpour ${ }^{\dagger}$ \\ Department of Chemistry, Faculty of Science, Ilam University, P.O. Box 69315516, Ilam, Iran \\ *E-mail: enikoorazm@yahoo.com (M. Nikoorazm), arashghch58@yahoo.com (A. Ghorbani-Choghamarani) \\ ${ }^{\dagger}$ Organic Polymer Chemistry Research Laboratory, Department of Chemistry, Isfahan University of Technology, Isfahan, Iran \\ Received April 23, 2010, Accepted June 7, 2010
}

Key Words: Guanidinium nitrate, Silica sulfuric acid, Urazole, Triazolinedione, Oxidation

Due to increasing concern about environmental impact, there has been tremendous recent interest in developing new processes that both reduce energy consumption and minimize pollution in chemical synthesis and processing. ${ }^{1}$ In the last few years, heterogeneous systems ${ }^{2-5}$ have become increasingly used in organic reactions, mainly because the reactions are carried out under mild conditions and the organic products are easily isolated from the reaction media. In addition, the stability and cheapness, high yields of the products, short reaction times, and reusability is other important advantages of these systems. ${ }^{6}$

4-Substituted-1,2,4-triazole-3,5-diones are of important class of five-member heterocyclic compounds, which can be prepared from the oxidation of urazoles and bis-urasoles. They have been used both as substrate and reagent in various organic reactions such as reaction of allylsilanes with triazolinedione, ${ }^{6}$ triazolinedione-alkene ene reaction, ${ }^{7}$ reaction of a triazolinedione with alkenes, ${ }^{8}$ reaction of triazolinedione with carbon nanotubes, ${ }^{9}$ oxidation of 1,4-dihydropyridines, ${ }^{10}$ oxidation of alcohols to aldehydes and ketones, ${ }^{11}$ oxidative coupling of thioles. ${ }^{12}$ High sensitivity and unusual reactivity of 1,2,4-triazole3,5-diones make them of interest to organic chemists, also make them difficult to prepare and purify. Therefore, finding oxidizing systems, which are safely and mildly able to convert urazoles to their corresponding triazolinediones it's of great importance.

In continuation of our studies on the application of new reagents in organic functional group transformations ${ }^{14-23}$ we became interested to introduce a metal-free catalytic media for the conversion of 4-substituted urazoles into triazolinediones by mixing of guanidinium nitrate and catalytic amounts of silica sulfuric acid.

Eventually we wish to report here a metal-free, catalytic and green procedure for the chemoselective oxidation of urazoles and bis-urazoles $\mathbf{1}$ to the corresponding triazolinediones $\mathbf{2}$ using guanidinium nitrate $\mathbf{I}$ and a catalytic amount of silica sulfuric acid $\mathrm{II}$ in the presence of wet $\mathrm{SiO}_{2}(50 \% \mathrm{w} / \mathrm{w}),($ Scheme 1 and<smiles>[Y]n1[nH]c(=O)n([X])c1=O</smiles>

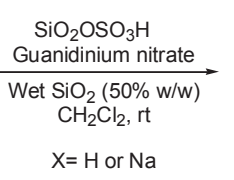<smiles></smiles>

Scheme 1
Table 1. Oxidation of urazole derivatives to the corresponding triazolinediones using guanidinium nitrate $\mathbf{I}$ and catalytic amounts of silica sulfuric acid II in the presence of wet $\mathrm{SiO}_{2}(50 \% \mathrm{w} / \mathrm{w})$ in dichloromethane at room temperature

Entry


Table 1. Continued

\begin{tabular}{llll}
\hline \multirow{2}{*}{ Entry Substrate $\quad$ Product } & $\begin{array}{l}\text { Substrate/ } \\
\text { Reagents/ } \\
\text { Catalyst }^{a}\end{array}$ & $\begin{array}{l}\text { Time Yield } \\
(\mathrm{min})\end{array}(\%)^{b}$ \\
\cline { 3 - 4 } & & I II & \\
\hline
\end{tabular}

7<smiles>O=c1[nH][nH]c(=O)n1C1CCCCC1</smiles><smiles></smiles>

$\begin{array}{lll}1.5 & 0.08 \quad 45\end{array}$

91<smiles>CCn1c(=O)[nH][nH]c1=O</smiles><smiles></smiles>

$\begin{array}{llll}1.5 & 0.08 & 155 & 90\end{array}$

10<smiles>CCCN1C(=O)NNC(=O)N1CC</smiles><smiles></smiles><smiles>CC(C)(C)n1c(=O)[nH][nH]c1=O</smiles><smiles></smiles><smiles>Cn1c(=O)[nH][nH]c1=O</smiles><smiles>CCCCCn1c(=O)[nH][nH]c1=O</smiles>
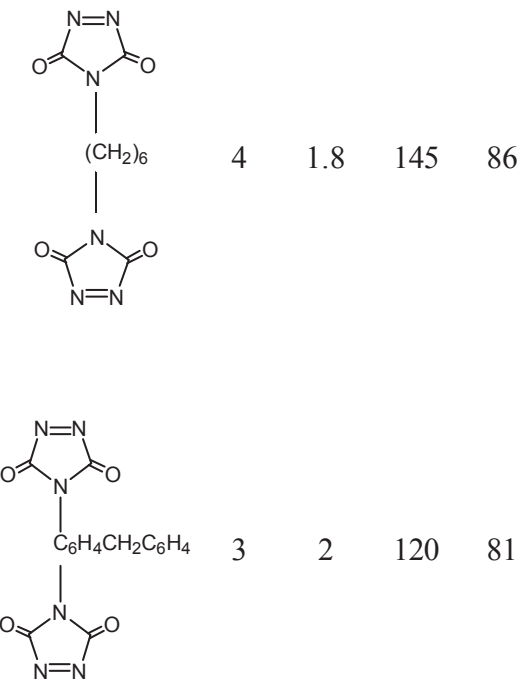
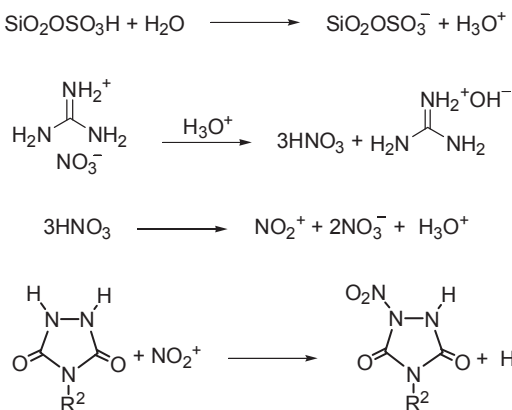<smiles>O=c1[nH]c([O-])c([18OH])[nH]1</smiles><smiles>O=C1NCCN1[N+](=O)[O-]</smiles>

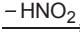<smiles>CCn1c(=O)nnn1-c1ccccc1</smiles>

Scheme 2

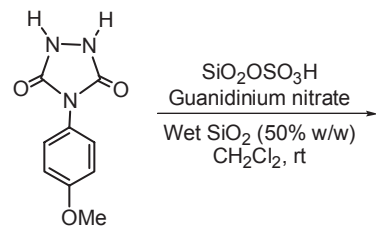<smiles>COc1ccc(N2C(=O)N=NC2=O)cc1</smiles><smiles></smiles>

Scheme 3

Table 1).

All reactions were carried out under mild and completely heterogeneous conditions at room temperature. Reaction components except pink-to-red color triazolinedione are insoluble in the reaction solvent. Consequently, 4-substituted-1,2,4-triazole-3,5-diones were prepared readily by mixing of a urazole, guanidinium nitrate, wet $\mathrm{SiO}_{2}(50 \% \mathrm{w} / \mathrm{w})$ and a catalytic amount of silica sulfuric acid; then stirring of this mixture at room temperature. Pure triazolinedion can be easily isolated from the reaction media by washing the reaction mixture with dichloromethane, simple filtration and evaporation of the solvent.

In order to show the catalytic role of silica sulfuric acid, 4phenyl-urazole was selected for the oxidation reaction in the absence of the catalyst, as typical example. Surprisingly, no triazolinedione was observed after 10 hours (Table 1, entry 2).

A plausible mechanism of this reaction is outlined in Scheme 2 based on previously reported works. ${ }^{21,27}$

The critical point of this mechanism is auto ionization of nitric acid, which generates in situ nitronium ion $\left(\mathrm{NO}_{2}{ }^{+}\right)$. Surprisingly, when a urazole with activated aromatic moiety (for example 4-methoxyphenyl urazole) was subjected to oxidation reaction with described system, 4-(4-methoxyphenyl)-1,2,4-triazole-3,5dione was selectively produced and no nitrated product was observed (Table 1 entry 5 and Scheme 3).

In summary herein we report a new catalytic procedure for the chemoselective oxidation of urazole derivatives to the corresponding triazolinediones under metal-free, mild and heterogeneous conditions. This method offers the advantage of shorter reaction times, high selectivity, non-toxic conditions, cost effective reagents and catalyst and easy workup.
${ }^{a} \mathrm{Wet}_{\mathrm{SiO}_{2}}:$ Substrate $=1 \mathrm{mmol}: 0.2 \mathrm{~g} ; \mathbf{I}$ refers to mmol of guanidinium nitrate and II refers to gram of silica sulfuric acid. ${ }^{b}$ Isolated yield. ${ }^{c}$ Reaction proceeds in the absence of silica sulfuric acid. 


\section{Experimental}

General. Chemicals were purchased from Fluka, Merck and Aldrich chemical companies. The oxidation products were characterized by comparison of their spectral (IR, ${ }^{1} \mathrm{H}$ NMR, and ${ }^{13} \mathrm{C}$ NMR) and physical data with authentic samples. ${ }^{24-26}$

Oxidation of 4-(4-methoxyphenyl)-urazole to 4-(4-methoxyphenyl)-1,2,4-triazoline-3,5-dione with guanidinium nitrate and silica sulfuric acid. A suspension of 4-(4-methoxyphenyl)urazole $(0.207 \mathrm{~g}, 1 \mathrm{mmol})$, guanidinium nitrate $(0.090 \mathrm{~g}, 1.5$ $\mathrm{mmol}), \mathrm{SiO}_{2}-\mathrm{OSO}_{3} \mathrm{H}(0.08 \mathrm{~g})$ and wet $\mathrm{SiO}_{2}(50 \% \mathrm{w} / \mathrm{w})(0.2 \mathrm{~g})$ in dichloromethane $(5 \mathrm{~mL})$ was stirred at room temperature for $60 \mathrm{~min}$ and then filtered. The residue was washed with $\mathrm{CH}_{2} \mathrm{Cl}_{2}$ $(20 \mathrm{~mL})$. Anhydrous $\mathrm{Na}_{2} \mathrm{SO}_{4}(2 \mathrm{~g})$ was added to the filtrate and filtered off after $20 \mathrm{~min}$. Finally $\mathrm{CH}_{2} \mathrm{Cl}_{2}$ was removed and 4-(4methoxyphenyl)-1,2,4-triazoline-3,5-dione was obtained in $98 \%$ yield $(0.201 \mathrm{~g}) .{ }^{1} \mathrm{H}$ NMR $\left(90 \mathrm{MHz}, \mathrm{CDCl}_{3}\right) \delta$ 7.28-7.39 $(\mathrm{d}, 2 \mathrm{H}), 6.97-7.07$ (d, 2H); IR (KBr) v 2971, 1770, 1604, 1515, $1408,1253,1177,1025 \mathrm{~cm}^{-1}$.

Acknowledgments. Financial support for this work by the Ilam University, Ilam, Iran is gratefully acknowledged.

\section{References}

1. Min, B. K.; Friend, C. M. Chem. Rev. 2007, 127, 2709.

2. Ghorbani-Choghamarani, A.; Zolfigol, M. A.; Ayazi-Nasrabadi, R. J. Braz. Chem. Soc. 2010, 21, 33.

3. Gao, Y.; Lam, Y. Adv. Synth. Catal. 2008, 350, 2937.

4. Zolfigol, M. A.; Bagherzadeh, M.; Niknam, K.; Shirini, F.; Mohammadpoor-Baltork, I.; Ghorbani-Choghamarani, A.; Baghbanzadeh, M. J. Iran. Chem. Soc. 2006, 3, 73.

5. Abraham, S.; Rajan, P. K.; Sreekumar, K. Polym. J. 1997, $29,12$.

6. Shirini, F.; Zolfigol, M. A.; Salehi, P.; Abedini, M. Curr. Org. Chem. 2008, 12, 183.

7. Wei, Y.; Lemal, D. M. Org. Lett. 2004, 6, 3837.

8. Vougioukalakis, G. C.; Roubelakis, M. M.; Alberti, M. N.; Orfanopoulos, M. Chem. Eur. J. 2008, 14, 9697.

9. Syrgiannis, Z.; Koutsianopoulos, F.; Muir, K. W.; Elemes, Y.
Tetrahedron Lett. 2009, 50, 277.

10. Menard-Moyon, C.; Gross, M.; Bernard, M.; Turek, P.; Doris, E.; Mioskowski, C. Eur. J. Org. Chem. 2007, 4817.

11. Zolfigol, M. A.; Ghorbani-Choghamarani, A.; Shahamirian, M.; Safaiee, M.; Mohammadpoor-Baltork, I.; Mallakpour, S. E.; Abdollahi-Alibeik, M. Tetrahedron Lett. 2005, 46, 5581.

12. Cookson, R. C.; Stevens, I. D. R.; Watts, C. T. Chem. Commun. 1966, 744.

13. Christoforou, A.; Nicolaou, G.; Elemes, Y. Tetrahedron Lett. 2006, 47, 9211.

14. Ghorbani-Choghamarani, A.; Goudarziafshar, H.; Nikoorazm, M.; Yousefi, S. Lett. Org. Chem. 2009, 6, 535.

15. Ghorbani-Choghamarani, A.; Rezaei, S. J. Chin. Chem. Soc. 2009, 56,251

16. Habibi, D.; Zolfigol, M. A.; Safaiee, M.; Shamsian, A.; GhorbaniChoghamarani, A. Catal. Commun. 2009, 10, 1257.

17. Amani, K.; Zolfigol, M. A.; Ghorbani-Choghamarani, A.; Hajjami, M. Monatsh. Chem. 2009, 140, 65.

18. Zolfigol, M. A.; Amani, K.; Hajjami, M.; Ghorbani-Choghamarani, A. Monatsh. Chem. 2008, 139, 895.

19. Zolfigol, M. A.; Amani, K.; Ghorbani-Choghamarani, A.; Hajjami, M.; Ayazi-Nasrabadi, R.; Jafari, S. Catal. Commun. 2008, 9, 1739.

20. Ghorbani-Choghamarani, A.; Chenani, Z.; Mallakpour, S. Synthetic Commun. 2009, 39, 4264.

21. Ghorbani-Choghamarani, A.; Goudarziafshar, H.; Nikoorazm, M.; Yousefi, S. Can. J. Chem. 2009, 87, 1144.

22. Ghorbani-Choghamarani, A.; Nikoorazm, M.; Goudarziafshar, H.; Tahmasbi, H. Bull. Korean. Chem. Soc. 2009, 30, 1388.

23. Ghorbani-Choghamarani, A.; Shiri, L.; Zeinivand, J. Bull. Korean Chem. Soc. 2008, 29, 2496.

24. Zolfigol, M. A.; Bagherzadeh, M.; Mallakpour, S.; Chehardoli, G.; Kolvari, E.; Ghorbani-Choghamarani, A.; Koukabi, N. Catal. Commun. 2007, 8, 256.

25. Zolfigol, M. A.; Ghorbani-Vaghei, R.; Mallakpour, S.; Chehardoli, G.; Ghorbani-Choghamarani, A.; Hosain-Yazdi, A. Synthesis 2006, 1631.

26. Zolfigol, M. A.; Chehardoli, G.; Ghaemi, E.; Madrakian, E.; Zare, R.; Azadbakht, T.; Niknam, K.; Mallakpour, S. Monatsh. Chem. 2008, 139, 261.

27. Ghorbani-Choghamarani, A.; Hajjami, M.; Goudarziafshar, H.; Nikoorazm, M.; Mallakpour, S.; Sadeghizadeh, F.; Azadi, G. Monatsh. Chem. 2009, 140, 607. 